\title{
REDUCCION DE UNA SINGULARIDAD A UNA SINGULARIDAD CON 1-JET NO NULO POR BLOWING-UPS DIRECCIONALES SUCESIVOS
}

\section{RIGOBERTO BELTRAN B , *}

\section{RESUREN .}

En este trabajo, se presentan las nociones de Blowing-ups direc cionales sucesivos $y$ de sucesión maximal de Blowing-ups de un campo de vectores $X$, en una dirección $D$, introducidas en [BO].

En primer lugar, se muestra que si la sucesión de Blowing-ups es finita entonces existe cono $\mathrm{K}$, de contacto finito, en torno de la direc ción $D$, tal que las órbitas entran en $\mathrm{K}$ Y abandonan $\mathrm{K}$ después de un tiempo finito; y si esta sucesión de Blowing-ups es infinita, entonces D es formalmente invariante bajo $\mathrm{x}$.

Finalmente, se muestra que si $x$ tiene grado de degeneración finito y que si $D$ es formalmente invariante después de un número finito de Blowing-ups en la dirección $D$, se obtiene un campo de vectores con 1-jet

* Académico Departamento de Matemáticas, Facultad de Ciencias. Universidad de Tarapacá, Arica - Chile. 
no nulo.

2. DEFINICIONES.

En este capítulo se introduce el vocabulario usual necesario pa ra el objeto principal de este trabajo. Se presenta el espacio de las sin gulariaades y las definiciones de los conceptos básicos.

Denotaremos por $\hbar^{k}\left(I R^{n}\right), I \leq k \leq \infty$ al conjunto de los campos de Vectores en $I R^{n}$, de clase $C^{k}$.

(2.1) Definición: Sea $X \varepsilon *^{k}\left(I R^{n}\right)$.

$x$ tiene una SINGULARIDAD en peIR $^{\mathrm{n}}$ si $\mathrm{X}(\mathrm{p})=0$.

Como solamente estaremos interesados en propiedades locales de una singularidad en un punto $p \varepsilon I R^{n}$, entonces podemos suponer, sin mayor restricción que $p=0$.

(2.2) Definición: Sean $X, Y \in z^{k}\left(I R^{n}\right)$

Diremos que $\mathrm{X}$ e $\mathrm{Y}$ SOn GERMEN-EQUIVALENTES en 0 si existe vecindad $\mathrm{U}$ de 0 tal que $\left.\mathrm{X}\right|_{\mathrm{U}}=\left.\mathrm{Y}\right|_{\mathrm{U}^{*}}$

Explícitamente, los campos $\mathrm{X}$ e $\mathrm{Y}$ son germen-equivalentes en 0 cuando existe vecindad $\mathrm{J}$ de 0 tal que $X(\mathrm{p})=\mathrm{Y}(\mathrm{p}) \forall \mathrm{p} \varepsilon U$.

La relación germen-equivalentes es una relación de equivalencia en el conjunto $d^{k}\left(I R^{n}\right)$. Así, para un campo dado, podemos formar la clase de equivalencia de este campo. la cual queda constituida por todos los cam pos de clase $c^{k}$, de $I R^{n}$, que son germen-equivalentes con él.

(2.3) Definición: Sea $X ; \star^{k}\left(I R^{n}\right)$.

El GERMEN de $x$ en 0 es el conjunto de los campos de vectores de clase $C^{k}$, de $I R^{n}$, que son germen-equiva- 
lentes con $X$ en 0 .

Como es fácil apreciar, el germen de $x$ en 0 no es otra cosa que la clase de equivalencia de $\mathrm{x}$ por la relación germen-equivalentes en 0.

Análogamente, se define el germen en 0 en funciones, difeomor fismos, ....

El germen en 0 de un conjunto $A \subseteq I R^{n}$ es el germen en 0 de su función característica $\chi_{\mathrm{A}}$.

Por simplicidad, usaremos la misma notación tanto para el germen de una aplicación (o de un conjunto) como para su representante.

Denotaremos por $G^{n}$ al conjunto de los gérmenes en 0 , de los campos de vectores de clase $C^{\infty}$ en $I^{n}$.

(2.4) Definición: Sean $X, Y \in G^{n} \quad y \quad k \in I N$.

Diremos que $X$ e $Y$ son k-JET-EQUIVALENTES si sus de rivadas en 0 , incluidas hasta las de orden $k$, son iguales.

Explicitamente, escogido un sistema de coordenadas $\mathrm{x}=\left(\mathrm{x}_{1}, \mathrm{x}_{2}, \ldots, \mathrm{x}_{\mathrm{n}}\right)$ en torno de 0 , entonces $\mathrm{si} \mathrm{X}$ e $\mathrm{Y}$ son $\mathrm{k}$-jet-equivalentes en 0 , se tiene que,

$$
\begin{aligned}
& D^{r} X(0)=D^{r} Y(0), \quad 0 \leq r \leq k, \text { donde } \\
& D^{D} X(0)=X(0), D^{O} Y(0)=Y(0) \quad y \\
& D^{r}=\frac{\partial^{r}}{\partial x_{1}^{r} \partial x_{2}^{r} \ldots x_{n}^{r} k} \text { con } \sum_{i=1}^{k} r_{i}=r
\end{aligned}
$$


O sea, para que dos campos $X$ e $Y$ sean $k$-jet-equivalentes, exigimos la coincidencia de los campos y de las derivadas parciales hasta las de orden $k$, en el punto 0 .

Así, el desarrollo en serie de Taylor en torno del punto 0 , de $X$ e $Y$, incluyendo los términos de grado $\leq k$ son idénticos.

La relación k-jet-equivalentes es una relación de equivalencia en el conjunto $\mathrm{G}^{\mathrm{n}}$.

(2.5) Definición: Sean $X \in G^{n} \quad y \quad k \in I N$.

El k-JET de $X$ es el conjunto de todos los $Y \in G^{n}$ que son $\mathrm{k}$-jet-equivalentes con $\mathrm{x}$ en 0 .

Como es făcil apreciar, el $\mathrm{k}$-jet de $\mathrm{x}$ es la clase de equivalencia de $x$ por la relación k-jet-equivalente.

Denotaremos por $j_{k} x(0)$ al $k$-jet del germen del campo $x$ en 0 .

Tomando los valores de las derivadas de $x$ en 0 comolas coor denadas del $k$-jet $j_{k} x(0)$, tenemos que $j_{k} x(0)$ es un espacio euclidiano. Así un k-jet puede ser visto como un conjunto de polinomios de grado $-k$ o por una n-tupla, $\alpha=\left(\alpha_{1}, \alpha_{2}, \ldots, \alpha_{N}\right)$ donde los $\alpha_{i}$ son polinomios de grado $\leq k$ para algún $\mathrm{N}$, función de $\mathrm{n} y \mathrm{k}$. Esto demuestra que existe una correspondencia biunivoca entre k-jets y campos de vectores $X$ de $I R^{n}$ con $X(0)=0$, cuyas componentes son polinomios de grado $\leq k$.

Esta correspondencia induce en el conjunto $\mathrm{J}_{\mathrm{k}}^{\mathrm{n}}$ de los $\mathrm{k}$-jet $j_{k} X(0)$ de $X \in G^{n}$ una estructura natural de espacio vectorial real.

Un sistema de coordenadas de $J_{k}^{n}$ es constituido por los valores de las derivadas parciales, hasta de orden $k$, calculadas en el pun to 0 . 
De esta manera, tenemos para cada k, una proyección canónica,

$$
\begin{aligned}
j_{k}: G^{n} & \longrightarrow J_{k}^{n} \\
x & \longrightarrow j_{k} x(0)
\end{aligned}
$$

la cual asocia a cada germen de un campo su k-jet en el origen.

gưn $X \& G^{n}$.

Es claro que todo $\sigma \varepsilon J_{k}^{n}$ es de la forma $\sigma=j_{k} \times(0)$ para al-

Estos espacios "k-jet" $J_{k}^{n}$ permiten poner una topología en $G^{n}$; la topología menos fina que torna las proyecciones $j_{k}$ continuas.

Ahora, notemos que si $k \leq r$ entonces, es claro que dos gérmenes que tengan el mismo $r$-jet en 0 , tendrán el mismo $k$-jet en 0 .

Así existe una proyección natural,

$$
\begin{aligned}
{ }_{r k}: & J_{r}^{n} \\
{ }_{r} x(0) & \longrightarrow J_{k}^{n}
\end{aligned}
$$

Esto es, " ${ }_{r k}$ queda definido por el "truncamiento" de los térmi nos de mayor orden. Estas funciones $\pi_{r k}$ son evidentemente sobreyectoras pero $\pi_{r k}$ no es inyectora excepto cuando $r=k$.

Además, como $\pi_{r k} \circ \pi_{m r}=\pi_{m k}$ para $m \geq r \geq k$ y $\pi_{r r}=i d$ $\forall r$, entonces podemos definir el límite inverso de los conjuntos $\mathrm{J}_{r}^{\mathrm{n}}$ por las funciones "rk.

Entonces, $J_{\infty}^{n}$ es el limite inverso (Proyectivo),

$$
J_{\infty}^{n}=1 \text { im } J_{r}^{n}
$$


También la función,

$$
\begin{aligned}
j_{\infty}: G^{n} & \longmapsto J_{\infty}^{n} \\
x & \longmapsto j_{\infty} x(0)
\end{aligned}
$$

es el límite inverso, $j_{\infty}=\lim j_{k}$

Estos elementos $j_{\infty} x(0)$ de $J_{\infty}^{n}$ son 1 lamados $\infty-J E T$.

En coordenadas locales, $j_{\infty} x(0)$ representa la serie de Taylor de $x$ en o. Así los elementos de $J_{\infty}^{n}$ pueden ser vistos como $n$-tuplas de series de Potencias en n-variables.

El Teorema de Borel: " $\forall T \in J_{\infty}^{n}$, $A X \varepsilon G^{n}$ tal que $T=j_{\infty} X(0)$ "; garantiza que $j_{\infty}$ es sobreyectora, lo cual establece que todo elemento de $J_{\infty}^{n}$ puede ser obtenido como ${ }^{n-j e t ~ d e l ~ g e r m e n ~ d e ~ a l g u ́ n ~ c a m p o ~ d e ~ v e c t o r e s . ~}$

Las funciones,

$$
\begin{aligned}
\pi_{k}: & J_{\infty}^{n} \longrightarrow J_{k}^{n} \\
j_{\infty} x(0) & \longmapsto j_{k} x(0)
\end{aligned}
$$

inducen en $J_{\infty}^{n}$ la topología menos fina que torna continuas las proyeccio nes $\pi_{k}$. Esta elección implica que $j_{\infty}: G^{n} \longrightarrow J_{\infty}^{n}$ es continua.

De manera análoga, se definen jets de funciones, difeomorfismos, ...

(2.5) Definición: Sean $X \in G^{n} \quad y \quad k \in I N$.

Diremos que $x$ tiene GRADO DE DEGENERACION $k$, si $j_{k} X(0)=0 \quad y \quad j_{k+1} x(0) \neq 0 \quad y$ tiene GRADO DE DEGENE RACION CERO si $j_{1} x(0) \neq 0$. 
(2.6) Definición: Una DIRECCION en 0 , es la imagen por un difeomorfismo de clase $C^{\infty}$ del germen en 0 de una semi-rectacon extremo en 0 .

El difeomorfismo es supuesto preserva el 0.

Es fácil probar que $s i \mathrm{D}$ es una dirección en $I R^{n}$, entonces existe germen de la aplicación $\gamma:(10, \infty), 0) \longrightarrow\left(\operatorname{IR}^{n}, 0\right)$ de clase $C^{\infty}$ con $\gamma^{\prime}(0) \neq 0$ tal que la imagen de $\gamma$ es $D$.

Recíprocamente, si $\gamma:([0, \infty), 0) \longrightarrow\left(I^{n}, 0\right)$ es de clase $c^{\infty}$ y si $\gamma^{\prime}(0) \neq 0$, entonces la imagen de $\gamma$ es una dirección.

(2.7) Definición: Sean $X \in G^{n} \quad y \quad D$ una dirección.

Diremos que $x$ es NO-DEGENERADO en la dirección D si para algún germen de clase $c^{\infty}, \gamma:([0, \infty), 0) \longrightarrow$ $\left(I R^{n}, 0\right)$ con $\gamma^{\prime}(0) \neq 0$ e imágen $D$, tenemos que $j_{\infty}(\mathrm{X} \circ \gamma)(0) \neq 0$.

De otra forma, diremos que $\mathrm{x}$ es DEGENERADO en la direc ción D.

(2.8) Definición: Sea $X \in G^{n}$.

Diremos que $x$ satisface la DESIGUALDAD DE LOJASIEWICZ, si existe $k \varepsilon$ IN $y \quad c, \delta \varepsilon I^{+}$tal que, $\forall x \in I^{n}$ con ||$x|| \leq \delta,|| x(x)|| \geq C|| x||^{k}$

Una propiedad importante es que si $x \in G^{n}$ satisface la desigualdad de Lojasiewicz, entonces $x$ es no-degenerado a lo largo de toda dirección.

Denotemos por $(x, z)$ un elemento de $\operatorname{IR}^{\mathrm{Im}} \mathrm{x}$ IR $y$ por $\left(\mathrm{x}_{\mathrm{x}}, \mathrm{x}_{\mathrm{z}}\right)$ a un campo de vectores $x$ en $I R^{m} x$ IR.

(2.9) Definición: Sea $X \in G^{m+1}$. 
Diremos que $x$ deja el eje-z, $\{0\}^{\mathrm{m}} \mathrm{x}$ IR FORMALMENTE INVARIANTE si para todo $k \in I N$,

$$
\frac{\partial^{k} x_{x}(0)}{\partial z^{k}}=0
$$

Esto es lo mismo que decir que $j_{\infty} x_{x}(0)$ no contiene puros têrminos en $z$.

(2.10) Definición: Sea $K \subset I^{m+1}$.

Un germen de $k$ es un CONO DE CONTACTO $k, k \varepsilon I N$, $\mathrm{k}>1$, de clase $\mathrm{C}^{1}$ si existe germen de una función

$h:((0, \infty), 0) \longrightarrow(I R, 0)$ de clase $c^{1}$ con $j_{k} h(0)=$ $0, j_{k+1} h(0) \neq 0$ y germen de un difeomorfismo, $\psi:\left(I R^{m+1}, 0\right) \longrightarrow\left(I R^{m+1}, 0\right)$ tal que, $K=\varphi\left(\left\{(x, z) \varepsilon I^{m} x[0, \infty) /|| x||<h(z)\right\}\right)$

$\mathrm{K}$ es 1 lamado un Cono de Contacto $\mathrm{k}$, en la dirección $D=\psi\left(\{0\}^{\mathrm{m}} \times[0, \infty)\right)$.

Mayores detalles y propiedades de los elementos definidos se pue den encontrar en la Bibliografía.

Como se dice al principio, este capítulo solamente está destina do a entregar el lenguaje básico a usar.

\section{BLOWING-UPS DE CAMPOS DE VECTORES.}

El Método de Blowing-up para Campos de vectores es una conocida têcnica que permite descomponer una singularidad en otras más simples. Este Método fue introducido por GOMORY en 1955 y por NEMYTSKII y STEPANOV en 1960 y ha sido usado, entre otros por TAKENS [Ta] y DUMORTIER [Du]. 
En este capítulo se presenta una descripción del Método y en par ticular, para Blowing-up en una dirección, se dan algunos cálculos especí ficos en forma detallada.

\subsection{UN BLOWING-UP ESFERICO.}

$$
\begin{aligned}
& \text { Para } m \in I N \text {, escribiremos, } \\
& s^{m}=\left\{\left(x_{1}, x_{2}, \ldots, x_{m+1}\right) \in I R^{m+1} / \sum_{i=1}^{m+1} x_{i}^{2}=1\right\} \\
& y \quad \phi: s^{m} x I R \longrightarrow I R^{m+1} \\
& \quad(x, r) \longrightarrow r x
\end{aligned}
$$

Observemos que:

$$
\begin{aligned}
& \text { 1. } \phi_{S^{m}{ }_{X I R^{+}}} \text {es un difeomorfismo de clase } C^{\infty} \text { sobre } I^{m+1}-\{0\} \\
& \text { 2. } s^{m} x\{0\}=\phi^{-1}(0)
\end{aligned}
$$

(3.1) Proposición [Ta]

$$
\begin{aligned}
& \text { Sea } x \in \tilde{X}^{k}\left(I R^{m+1}\right) \text { con } x(0)=0 . \\
& \text { Entonces existe campo de vectores } \tilde{x} \text {, de clase } c^{K-1} \text { de- } \\
& \text { finido sobre } S^{m} x \text { IR tal que para todo } q \in S^{m} x I R, \\
& d \phi(\tilde{x}(q))=x(\phi(q)) \quad\left(0 \quad \phi_{*} \tilde{x}=x\right) .
\end{aligned}
$$

Del desarrollo en Serie de Taylor de $x$ en 0 , se observa que si $x$ tiene su 1-jet nulo, entonces $x$ es idénticamente nulo en $s^{m} x\{0\}$.

Ahora, si X tiene grado de degeneración $s$, entonces, por construcción, $\tilde{x}_{\mid} m_{x\{0\}}=0$, luego tiene sentido definir el campo de vec tores $\overline{\mathrm{x}}=\frac{1}{\mathrm{~s}^{\mathrm{s}}} \tilde{\mathrm{x}} \mathrm{S}_{\mathrm{S}}^{\mathrm{m}} \mathrm{x}\{0\}$ tomar el límite cuando $\mathrm{r} \longrightarrow 0$ obteniendo así un campo $r^{\mathrm{S}}$ de vectores de clase $c^{\mathrm{k-s-1}}$ en $\mathrm{s}^{\mathrm{m}} \mathrm{x}$ IR. 
(3.2) Definición: $\overline{\mathrm{x}}$ es 1 lamado el Campo de Vectores obtenido por un BLOW ING-UP y dividido por $r^{\mathrm{S}}$.

Notemos que $\bar{x}$ restricto a $S^{m} x\{0\}$ es el campo veçtorial tangente a $s^{\mathrm{m}} \times\{0\}$.

El estudio de este campo, a veces nos da información del compor tamiento asintótico de las órbitas cuando ellas tienden a 0.

\subsection{UN BLOWING-UP DIRECCIONAL.}

En dimensiones superiores, el cálculo específico para un Blowing-up esférico es complicado.

Restrinjamos nuestra atención a la semi-esfeca de $\mathrm{s}^{\mathrm{m}}$ y supongamos que tiene el punto $(0,0, \ldots 0,1)$ como "polo norte". Un Blowing-up direccional no es nada más que escoger una carta de $\mathrm{s}^{\mathrm{m}}$ usando proyeci ión central, con centro en 0 , en el hiperplano tangente por $(0,0, \ldots, 0,1)$ a $s^{m}$.

Denotemos por $(x, z)$ a un punto de $I^{m} x$ IR.

Entenderemos por eje-z al conjunto $\{0\}^{\mathrm{m}} \mathrm{x}$ IR.

para $n \in I N$, sea la aplicación,

$$
\begin{aligned}
\psi^{n}: I R^{m} \times I R & \longmapsto I R^{m} \times I R \\
(x, z) & \longmapsto\left(x z^{n}, z\right)
\end{aligned}
$$

Análogamente a la proposición (3.1), tenemos,

(3.3) Proposición: Sea $x \in \mathcal{y}_{0}^{k}\left(I R^{m} x\right.$ IR) con $x(0)=0$.

Entonces existe campo de vectores $\tilde{x}^{1}$ de clase $c^{k-1}$

en $I R^{m} \times I R$ tal que para todo $q \varepsilon I R^{m} \times I R$,

d $\psi^{1}\left(\tilde{x}^{1}(q)\right)=x\left(\psi^{1}(q)\right) \quad\left(0 \quad \psi_{*}^{1} \tilde{x}^{1}=x\right)$ 
Nuevamente, si $x$ tiene grado de degeneración $s$, definimos el campo de vectores,

$$
\bar{x}^{1}=\frac{1}{z^{s}} \tilde{x}^{1}
$$

y tomando el límite cuando $z \longrightarrow 0$, obtenemos un campos de vectores de clase $\mathrm{C}^{\mathrm{k-S}-1}$ en $\mathrm{IR}^{\mathrm{m}} \times \mathrm{IR}$.

(3.4) Definición: $\bar{x}^{-1}$ es llamado el campo de vectores obtenido por un BLOWING-UP EN LA. DIRECCION $z y$ dividido por $z^{s}$.

Denotando por $\mathrm{x}=\left(\mathrm{x}_{\mathrm{x}}, \mathrm{x}_{\mathrm{z}}\right)$ al campo de vectores $\mathrm{x}$ en $\operatorname{IR}^{\mathrm{m}} \mathrm{xIR}$, es fácil ver que,

$$
\begin{aligned}
\psi_{*}^{1} \tilde{x}^{1} & =x \quad \text { si y solamente si, } \\
\tilde{x}^{1} & =\left(\frac{1}{z}\left(x_{x} \circ \psi^{1}\right)-\frac{x}{z}\left(x_{z} \circ \psi^{1}\right), x_{z} \circ \psi^{1}\right) \\
\text { y asi, } \quad \bar{x}^{1} & =\left(\frac{1}{z^{s}}\left(\frac{1}{z}\left(x_{x} \circ \psi^{1}\right)-\frac{x}{z}\left(x_{z} \circ \psi^{1}\right)\right), \frac{1}{z^{s}}\left(x_{z} \circ \psi^{1}\right)\right)
\end{aligned}
$$

\subsection{RELACION ENTRE BLOWING-UPS DIRECCIONALES Y ESFERICOS.}

Denotemos por $s_{+}^{m}$ la "semi-esfera superior", esto es,

$$
s_{+}^{m}=\left\{\left(x_{1}, \ldots, x_{m^{\prime}}, z\right) \varepsilon s^{m} / z>0\right\}
$$

Consideremos la biyección,

$$
\begin{aligned}
f: S_{+}^{\mathrm{m}} \times I R & \longrightarrow I R^{\mathrm{m}+1} \\
& \left(\mathrm{x}_{1}, \ldots, \mathrm{x}_{\mathrm{m}^{\prime}}, \mathrm{r}\right) \longrightarrow\left(\frac{\mathrm{x}_{1}}{\mathrm{z}}, \ldots, \frac{\mathrm{x}_{\mathrm{m}}}{\mathrm{z}}, \mathrm{zr}\right)
\end{aligned}
$$


Entonces el siguiente diagrama conmuta,

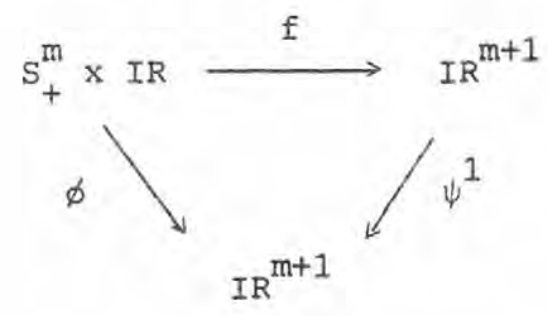

Luego, $\tilde{x}_{S_{+}^{m} x \text { IR }}$ y $\tilde{X}^{1}$ son difeomorfos $y$

$$
f_{*} \tilde{x}=\tilde{x}^{1}
$$

Si denotamos,

$$
f\left(x_{1}, \ldots, x_{m}, z, r\right)=\left(\frac{x_{1}}{z}, \ldots, \frac{x_{m}}{z}, z r\right)=\left(\bar{x}_{1}, \ldots, \bar{x}_{m}, \bar{z}\right)
$$

entonces, usando el hecho que,

$$
\sum_{i=1}^{m} x_{1}^{2}+z^{2}=1
$$

tenemos que $z=\frac{1}{\sqrt{1+\bar{x}_{1}^{2}+\ldots+\bar{x}_{m}^{2}}} \quad y \quad r=\frac{\bar{z}}{z}$.

Entonces, $\quad f_{\star} \bar{x}=f\left(\frac{1}{r^{S}} \tilde{X}\right)=\frac{z^{S}}{z^{S}} f_{*} \tilde{x}=\frac{z^{S}}{z^{S}} \tilde{X}^{1}=z^{S} \bar{X}^{1}$, de

donde, $\quad f_{*} \bar{x}=\frac{1}{\left(1+\bar{x}_{1}^{2}+\ldots+\bar{x}_{m}^{2} s / 2\right.} \bar{x}^{1}$

Como $\left(1+x_{1}^{2}+\ldots+x_{m}^{2}\right)^{-s / 2}$ es siempre positivo, las órbitas de $\bar{x}^{-1}$ $y f_{*} \bar{x}$ son las mismas y con la misma orientación. 
Así, el Blowing-up direccional es el mismo Blowing-up esférico, pero restringido a un pequeño dominio y transformado por un cambio local de coordenadas.

3.4. EL $\infty$-JET DE $\bar{x}^{1}$ EN 0 (Caso $I R^{m+1}$ )

Para $\alpha=\left(\alpha_{1}, \alpha_{2}, \ldots, \alpha_{m}\right) \in I N^{m}, \underset{m}{\operatorname{escribamos}}|\alpha|=\sum_{i=1}^{m} \alpha_{i}$
$x=\left(x_{1}, \ldots, x_{m}\right) \varepsilon I R^{m}$, denotemos $x^{\alpha}=\prod_{i=1}^{m} x_{i}^{\alpha} ;$
también $\frac{\partial^{|\alpha|}}{\partial x^{\alpha}}=\frac{\partial|\alpha|}{\partial x_{1}^{\alpha I} \ldots \partial x_{m}^{\alpha m}}$ y $\quad \alpha !=\prod_{i=1}^{m}\left(\alpha_{i} !\right)$.

Entonces, podemos escribir el $\infty$-jet de $x$ en 0 como

$j_{\infty} x(0)=\sum_{i=1}^{m} \sum_{n=s+1}^{\infty} \sum_{|\alpha|=0}^{n} a_{i, \alpha}^{n} x^{\alpha} z^{n-|\alpha|} \frac{\partial}{\partial x}+\sum_{i}^{\infty} \sum_{n=s+1}^{n} \sum_{|\alpha|=0}^{n} c^{n} x^{\alpha} z^{n-|\alpha|} \frac{\partial}{\partial z}$

si $x$ tiene grado de degeneración $s$ en 0 .

Para el $\infty$-jet de $\bar{x}^{1}$ en 0 , escribimos,

$j_{\infty} \bar{x}^{-1}(0)=\sum_{i=1}^{m} \sum_{n=0}^{\infty} \sum_{|\alpha|=0}^{n} a_{i, \alpha}^{-n} x^{\alpha} z^{n-|\alpha|} \frac{\partial}{\partial x_{i}}+\sum_{n=0}^{\infty} \sum_{|\alpha|=0}^{n} c_{\alpha}^{-n} x^{\alpha} z^{n-|\alpha|} \frac{\partial}{\partial z}$

Utilizando $(\Delta)$ y haciendo los cálculos se encuentra que:

1. $a_{i, \alpha}^{-n}=a_{i, \alpha}^{n+s+1-|\alpha|}-c_{\left(\alpha_{1}, \ldots, \alpha_{i-1}, \alpha_{1}-1, \alpha_{i+1}, \ldots, \alpha_{m}\right)}^{n+s+1-|\alpha|}$

para $1 \leq i \leq m \quad, \quad 1 \leq \alpha_{i}, \quad 1 \leq|\alpha| \leq n$

2. ${ }_{i,\left(\alpha_{1}, \ldots, \alpha_{i-1}, 0, \alpha_{i+1}, \ldots, \alpha_{m}\right)}^{n}=a_{i, \alpha}^{n+s+1-|\alpha|}$

para $1 \leq i \leq m, \quad 0 \leq|\alpha| \leq n$ 
3. $\vec{c}_{u}^{n}=c_{\alpha}^{n+s-|\alpha|}$, para $0 \leq|\alpha| \leq n$

En este caso, podemos ver que para cada $n \in I N$ y para cada

(4) con $x=\mathrm{n} ; \overrightarrow{\mathrm{c}}_{\alpha}^{-\mathrm{n}}=0$. Esto expresa el hecho que el hiperplano $z=0$ es invariante bajo $\bar{x}^{1}$, equivalentemente que la componente $\frac{\partial}{\partial z}$ de $\bar{x}^{-1}$ to contiene puros términos $x^{\alpha}$.

En 1., 2., 3., podemos tomar el miembro derecho igual a cero cuando son indefinidos.

\subsection{BLOWING-UP DIRECCIONAILBS SUCESIVOS.}

Analizando una singularidad, puede ocurrir que después de un Hlowing-up se encuentre un número de singularidades no suficiente simples a ser tratadas por los métodos conocidos. En estas singularidades podemos aplicar de nuevo un Blowing-up.

Sea $\bar{x}^{-1}$ obtenido por un Blowing-up a $x$ en la dirección-z $y$ uividido por $z^{s}$.

Supongamos que $\bar{x}^{-1}$ tiene de nuevo una singularidad en, digamos $\{a, 0\rangle \in \operatorname{IR}^{m} \times\{0\}$ y que $j_{p} \bar{x}^{-1}(a, 0)=0$ y $j_{p+1} \bar{x}^{-1}(a, 0) \neq 0$. Entonces podemos calcular el Blowing-up a $\bar{x}^{-1}$ en $(a, 0)$, en la dirección-z, de la siguiente manera natural:

Sea $T: I R^{m} \times I R \longrightarrow I R^{m} \times I R$

$$
(\mathrm{x}, \mathrm{z}) \longrightarrow(\mathrm{x}-\mathrm{a}, \mathrm{z})
$$

Como $\mathrm{T}_{\star} \overline{\mathrm{X}}^{-1}$ tiene una singularidad con grado de degeneración $\mathrm{p}$ en 0 , entonces podemos considerar el campo de vectores $\overline{T_{\star} \bar{X}^{1}}$ obtenido de $T_{*} \bar{X}^{1}$ por blowing-up, en la dirección-z y dividido por $z^{p}$. 
(3.5) Definición: ${\overline{\mathrm{T}} \overline{\mathrm{X}}^{1}}^{1}$ es el campo de vectores obtenido por DOS BLOWING-UPS EN LA DIRECCION-z, primero en $(0,0)$ y segundo en $(a, 0)$ y dividido primero por $z^{\text {s }}$ y luego por $z^{p}$.

de $\bar{x}^{-1}$.

Denotaremos a $\bar{T}_{*} \bar{X}^{1}$ por $\overline{\mathrm{X}}^{2}$.

Evidentemente, $\overline{\mathrm{x}}^{2}$ depende de la elección de la singularidad

De este modo, podemos construir sucesivamente $\bar{x}^{n}$ por Blowingup sobre $\overrightarrow{\mathrm{x}}^{\mathrm{n}-1}$ en alguna singularidad. Obviamente, $\overrightarrow{\mathrm{x}}^{\mathrm{n}}$ depende de la elección de las singularidades en las cuales hacemos el Blowing-up.

(3.6) Definición: Sea $x \in \frac{\pi}{x}^{\infty}\left(I R^{m} x I R\right)$ con $x(0)=0$.

Llamaremos de BLOWING-UPS DIRECCIONALES SUCESIVOS de $\mathrm{X}$, a una sucesión de triples $\left(\bar{x}^{n},\left(x_{n}, 0\right), s_{n}\right) 0 \leqslant n<N$

$N \in$ IN tal que:

1. $\overrightarrow{\mathrm{x}}^{0}=\mathrm{x} \quad \mathrm{y} \quad\left(\mathrm{x}_{0}, 0\right)=(0,0)$

2. $\forall \mathrm{n}, 0 \leq \mathrm{n}<\mathrm{N} ; \overline{\mathrm{x}}^{\mathrm{n}}$ tiene una singularidad con grado de degeneración $s_{n}$ en $\left(x_{n}, 0\right)$ y $\bar{x}^{n+1}$ es obtenido por Blowing-up a $\bar{x}^{n}$ en $\left(x_{n}, 0\right)$ y dividi do por $z^{S} n$.

A continuación, se encuentran algunas fórmulas explícitas para Blowing-ups direccionales sucesivos en 0 .

(3.7) Lema: Sea $\bar{x}^{n}$ obtenido por Blowing-ups direccionales sucesivos en 0 , de $x$. Entonces,

$\overrightarrow{\mathrm{x}}=\frac{1}{\mathrm{z}^{\mathrm{s}} \mathrm{s}_{2}+\ldots+\mathrm{s}_{\mathrm{n}-1}} \tilde{\mathrm{x}}^{\mathrm{n}}$, donde $\tilde{\mathrm{x}}^{\mathrm{n}}$ tiene la propiedad $\psi_{*}^{n}\left(\tilde{x}^{n}(q)\right)=x\left(\psi^{n}(q)\right), \quad \forall q \in I^{m} x I R$ 
Como en $(\Delta)$ tenemos que para $x=\left(x_{x}, x_{z}\right), \quad \psi_{\star}^{n \sim x} x^{n}=x$ si sola mente si,

$$
\begin{gathered}
\tilde{x}^{n}+\left(\frac{1}{z^{n}}\left(x_{x} \circ \psi^{n}\right)-\frac{n x}{z}\left(x_{z} \circ \psi^{n}\right), x_{z} \circ \psi^{n}\right) \\
\text { Así, } \quad \bar{x}^{n}=\frac{1}{z^{s} 0^{+s_{1}+\ldots+s_{n-1}}}\left(\frac{1}{z^{n}}\left(x_{x} o \psi^{n}\right)-\frac{n x}{z}\left(x_{z} \circ \psi^{n}\right), x_{z} o \psi^{n}\right)
\end{gathered}
$$

Cabe hacer notar que no existe restricción, por cambios de coor denadas $C^{\infty}$, suponer que los Blowing-ups direccionales sucesivos son en la dirección del eje-z. Se deduce de esto que existe correspondencia uno a uno entre sucesiones direccionales y $(\mathrm{N}-1)$-jet de direcciones (incluido $N=\infty$ ).

(3.8) Definición: Blowing-ups direccionales sucesivos de $x$ donde $\forall n$, $0 \leq n<N,\left(x_{n}, 0\right)=(0,0)$ serán llamados SUCESIONES DE BLOWING-UPS de $x$, en 0 , en la dirección $-z$. Denotaremos tal sucesión por $\left(\vec{x}^{n}, 0, s_{n}\right) 0 \leq n<N$

Ahora, si tenemos una sucesión de Blowing-ups de $\mathrm{x}$, a 10 largo del eje-z, es posible, después de un tiempo finito, no tener más singularidades en $(0,0)$. Esto podemos formalizarlo como sigue:

(3.9) Definición: La sucesión $\left(\bar{x}^{n}, 0, s_{n}\right) \leq n<N$ de Blowing-ups de $x$,

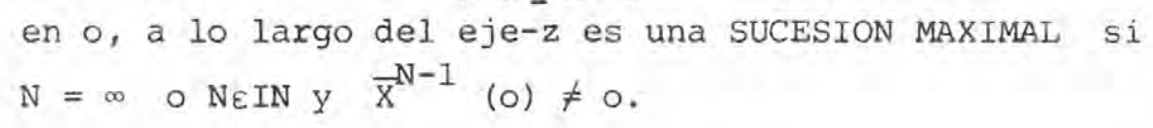

Sucesiones maximales de Blowing-ups de campo de vectores, siempre existen.

(3.10) Proposición: Sea $N \in I N,\left(\bar{x}^{n}, 0, s_{n}\right)_{0} \leq n<N \quad$ Sucesiónmaximal de Blowing-ups de $X$. Entonces existe cono $K$, de con 
tacto $\mathrm{N}-2$, en torno al eje-z tal que todas las órbitas en el interior de $\mathrm{K}$, entran en $\mathrm{K} y$ dejan $K$ después de un tiempo finito.

\section{Demostración :}

Como $\overrightarrow{\mathrm{X}}^{\mathrm{N}-1}(0) \neq 0$, podemos construir una vecindad en forma de cilindro, de $0 \operatorname{IR}^{m} \times[0, \infty)$, de la forma,

$$
C=\left\{(x, z) \varepsilon \operatorname{IR}^{m} x I R /|| x|| \leq R, z \varepsilon[0, \delta)\right\}
$$

tal que todas las órbitas de $\overrightarrow{\mathrm{x}}^{\mathrm{N}-1}$ en el interior de $\mathrm{C}$, entran en $C$ y dejan $C$ después de un tiempo finito.

Entonces,

$$
\begin{aligned}
\psi^{N-1}(C) & =\left\{\left(z^{N-1} x, z\right) /|| x|| \leq R, z \varepsilon[0, \delta)\right\} \\
& =\left\{\left(x^{\prime}, z\right) /|| x^{\prime}|| \leq z^{N-1} R, z \in[0, \delta)\right\}
\end{aligned}
$$

Sea $K$ el germen en 0 de $\psi^{N-1}(C)$.

Luego el resultado es inmediato.

Consideremos ahora sucesiones infinitas de Blowing-ups.

Cabe indicar que esto establece que la sucesión maximal de Blow ing-ups de un campo $x$, a lo largo del eje-z, es infinita.

(3.11) Proposición: Sea $\left(\overline{\mathrm{x}}^{\mathrm{n}}, 0, \mathrm{~s}_{\mathrm{n}}\right)_{0} \leq \mathrm{n}<\mathrm{N}$, sucesión maximal de Blowingups de $x$, a lo largo del eje-z.

Entonces $\mathrm{N}=\infty$ si, $\mathrm{y}$ solamente si, el eje-z es for malmente invariante bajo $\mathrm{x}$.

Demostración:

i) $\Longrightarrow$ Sea $j_{\infty} x(0)=\sum_{i=1}^{m} \sum_{S=s_{0}+1}^{\infty} \sum_{|\alpha|=0}^{s} a_{i, \alpha}^{s} x^{\alpha} s-|\alpha| \frac{\partial}{\partial x_{i}}+$ 


$$
\begin{aligned}
& +\sum_{S=s_{0}+1} \sum_{|\alpha|=0}^{S} c_{i, \alpha}^{S} x^{\alpha} z-|\alpha| \frac{\partial}{\partial z} \\
& j_{\infty} \vec{x}^{n}(0)=\sum_{i=1}^{m} \sum_{S=s_{n}+1}^{\infty} \sum_{|\alpha|=0}^{s} n a_{i, \alpha}^{S} x^{\alpha} z^{S-|\alpha|} \frac{\partial}{\partial x_{i}}+ \\
& +\sum_{S=s_{n}+1}^{\infty}{ }^{n-s} c_{i, \alpha}^{\alpha} x^{s-|\alpha|} \frac{\partial}{\partial z}
\end{aligned}
$$

Debemos probar que $a_{i, 0}^{S}=0 \quad \forall S \geq s_{0}+1$ e $i \varepsilon\{1, \ldots, m\}$

Con la fórmula 2. ( $\sec .3 .4)$ en encuentra que $\forall S \geq s_{0}+1$ e $i \varepsilon\{1, \ldots, m\}, \quad a_{i, 0}^{-s-s} 0^{-1}=a_{i, 0}^{s} y$ por inducción sobre $n$, usando la mis ma fórmula tenemos que $\forall \mathrm{n} \varepsilon \mathrm{IN}$,

$$
\mathrm{n}_{i, 0}^{n-s-\mathrm{o}^{\circ}}-\mathrm{s}_{n-1}-\mathrm{n}=a_{i, 0}^{s}
$$

Supongamos por contradicción, que $s_{i, 0}^{S}=0$. Entonces,

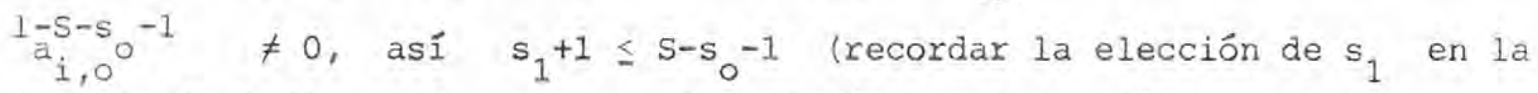
definición 3.6) o $0 \leq S-s_{0}-s_{1}-2$; además por inducción encontramos,

$$
\mathfrak{a}_{i, 0}^{n-s-s_{0}}-s_{1}-\cdots s_{n-1}^{-n} \neq 0
$$

como siempre, como debiera ser, $0 \leq \mathrm{s}-\mathrm{s}_{0}-\cdots-\mathrm{s}_{\mathrm{n}-1} \mathrm{n}$.

Ciertamente, tarde $\circ$ temprano $0=s-s_{0}-\cdots-s_{n-1} n$ para algún $n$. $N=\infty$. Pero, $\bar{x}^{n}(0)=\sum_{i=1}^{m} n_{i, 0}^{n-0} \frac{\partial}{\partial x_{i}} \neq 0$, contradiciendo el hecho que ii) U Usando la misma notación, tenemos que $a_{i, 0}^{S}=0, \forall s \geq s_{0}+1$ e 
i $\varepsilon\{1, \ldots, m\}$. La fórmula 2 . de la secc. 3.4 , da inmediatamente que,

$$
\stackrel{n-0}{a, 0}_{i, 0}=0 \forall n \in I N \quad \text { e } i \in\{1, \ldots, m\}
$$

de donde, $\bar{x}^{n}(0)=0 \forall n$.

Luego, de i) y ii), se tiene el resultado.

\section{REDUCCION A URA SINGULARIDAD CON 1-JET NO NULO.}

El propósito de esta sección es probar el siguiente

TEOREMA: Sea $\left(\overline{\mathrm{x}}^{\mathrm{n}}, 0, \mathrm{~s}_{\mathrm{n}}\right)_{0} \leq \mathrm{n}<\mathrm{N}$ sucesión maximal de Blowing-ups de $X \in G^{m+1}$ a lo largo del eje-z $\{0\}^{m} x[0, \infty)$. Si $X$ es no degene rado a lo largo del eje-z entonces existe $N \varepsilon$ IN tal que $\overrightarrow{\mathrm{x}}^{\mathrm{N}+\overline{\mathrm{n}}}$ tiene grado de degeneración cero $\forall \mathrm{n} \varepsilon \mathrm{IN}$.

La demostración de este Teorema será consecuencia de las proposiciones (4.1), (4.2), (4.3), (4.4), (4.5) y (4.6) que se verán a continuación.

(4.1) Proposición: Sea $X \varepsilon G^{m+1}$ con grado de degeneración $s$. Si $\bar{x}^{-1}$ tiene grado de degeneración $\geqslant s+1$, entonces $j_{s+1} x(0)=\sum_{|\alpha|=s+1} c_{\alpha}^{s+1} x^{\alpha} \frac{\partial}{\partial z}$.

Demostración :

$$
\begin{aligned}
& \text { Como } j_{s} x(0)=0 \text {, solamente tenemos que ver } a_{i, \alpha}^{s+1} \text { y } \\
& c_{\alpha}^{s+1}, \quad 0 \leq|\alpha| \leq s+1,1 \leq i \leq m \text {. } \\
& \text { De la fórmula } 3 \text {. secc. } 3.4 \text {, tenemos que para cada } n \text { con } \\
& 1 \leq n \leq s \text { y con }|\alpha|=n-1,0=c^{-n}=c_{\alpha}^{n+s-n-1}=c_{\alpha}^{s+1} \text {. } \\
& \text { Así, } c_{\alpha}^{s+1}=0 \text { para cada } \alpha \text { con } 0 \leq|\alpha| \leq s(\nabla) \text {. }
\end{aligned}
$$


De la parte 1. de las fórmulas de la secc. 3.4, tenemos que para cada $n$ con $1 \leq n \leq s+1$ con $|\alpha|=n$ y $1 \leq \alpha_{i}$ $0=a_{i, \alpha}^{-n}=a_{i, \alpha}^{n+s+1-n}-c_{\left(\alpha_{1}, \ldots, \alpha_{i-1}, \alpha_{i}-1, \alpha_{i+1}, \ldots, \alpha_{m}\right)}^{n+s+1-n}$ $=a_{i, \alpha}^{s+1}-c_{\left(\alpha_{1}, \ldots, \alpha_{i-1}, \alpha_{i}-1, \alpha_{i+1}, \ldots, \alpha_{m}\right)}^{s+1}$

Pero por $(\nabla)$ anterior, estos $c$ son cero.

En consecuencia, $\quad c_{i, \alpha}^{s+1}=0$ para cada $\alpha$ con $0 \leq|\alpha| \leq s+1$ y $\quad 1 \leq \alpha_{i}$.

De la misma forma, usando 2. de la secc. 3.4 se obtiene que todos los coeficientes en $j_{S+1} X(0)$ se anulan, excepto, posi blemente, $c_{\alpha}^{s+1}$ con $|\alpha|=s+1$.

(4.2) Proposición: Si $\mathrm{X} \in \mathrm{G}^{\mathrm{m}+1}$ y si $\overline{\mathrm{X}}^{-1}$ tiene grado de degeneración $\mathrm{s}$, entonces $\overline{\mathrm{x}}^{2}$ tiene grado de degeneración $\leqslant s$.

\section{Demostración :}

Supongamos que $j_{s+1} \bar{x}^{2}(0)=0$.

Por la proposición (4.1) anterior, esto implica que,

$$
j_{s+1} \bar{x}^{-1}(0)=\sum_{|\alpha|=s+1}^{\sum} \bar{c}_{\alpha}^{-s+1} x^{\alpha} \frac{\partial}{\partial z}
$$

Por nuestro supuesto, uno de $\operatorname{los} \mathrm{c}_{\alpha}^{-\mathrm{s}+1}$, debe ser diferente de cero.

Esto es imposible por la observación en 3. secc. 3.4. (Inva riancia del hiperplano $z=0$ ) 
(4.3) Proposición: Si $X \in G^{m+1}$ tiene grado de degeneración $s$ y $j_{s+1} \bar{x}^{1}(0)=0$, entonces $\bar{x}^{-1}$ tiene grado de degenera ción s+1.

\section{Demostración :}

Debemos probar que $j_{s+2} \bar{x}^{-1}(0) \neq 0$.

De la Proposición (4.1), y del hecho que $j_{s+1} \bar{x}^{-1}(0)=0$, tenemos que

$$
j_{s+1} x(0)=\sum_{|\alpha|=s+1} c_{\alpha}^{s+1} x^{\alpha} \frac{\partial}{\partial z}
$$

Como $\mathrm{x}$ tiene grado de degeneración $s$, existe $\alpha$ con $|\alpha|=s+1 \quad$ y $\quad c_{\alpha}^{s+1} \neq 0$.

Usando 3. de la secc. 3.4, se observa que,

$$
c_{\alpha}^{-s+2}=c_{\alpha}^{s+2+s-s-1}=c_{\alpha}^{s+1}
$$

$$
\text { Asi, } j_{s+2} \bar{x}^{-1}(0) \neq 0 \text {. }
$$

(4.4) Proposición: Sea $X \in G^{m+1}$. Supongamos existe un entero $s \geqslant 1$ y una sucesión infinita $\left(\overline{\mathrm{x}}^{\mathrm{n}}, 0, \mathrm{~s}\right)_{n} \varepsilon$ IN de Blowingups de $x$, en 0 , en la dirección- $z$ tal que $\forall \mathrm{n} \in \mathrm{IN}, \overline{\mathrm{x}}^{\mathrm{n}}$ tiene grado de degeneración $\mathrm{s} y$ en cada paso dividimos por $\mathrm{z}^{\mathrm{S}}$.

Denotando por $i: I R \longrightarrow I R^{m+1}$

$$
z \longmapsto(0, \ldots, 0, z)
$$

entonces $j_{\infty}\left(j_{s-1}(x \circ i)\right)(0)=0$. 
Demostración:

$$
\begin{aligned}
& j_{S-1} x(0, \ldots, 0, z) \text { es formalmente determinado por } \\
& \begin{aligned}
\left.\frac{\partial^{M} x}{\partial x^{\beta} \partial z^{M-|\beta|}}(0, \ldots, 0, z)\right)_{0} \leq M \leq s-1 \\
0 \leq|B| \leq M
\end{aligned}
\end{aligned}
$$

Usando las notaciones de 3.4 ,

$$
\begin{aligned}
\frac{\partial^{M} x}{\partial x^{\beta} \partial z^{M-}|\beta|}\left(x_{1}, \ldots, x_{m}, z\right)= & \sum_{n=s+1}^{\infty} \sum_{\alpha \mid=0}^{n}\left(a_{1, \alpha}^{n} \frac{\partial}{\partial x_{1}}+\ldots+a_{m, \alpha}^{n} \frac{\partial}{\partial x_{m}}+c_{\alpha}^{n} \frac{\partial}{\partial z}\right) . \\
& \frac{\alpha !}{(\alpha-\beta)} x^{\alpha-\beta} \frac{(n-|\alpha|) !}{(n-|\alpha|-M+|\beta|) !} z^{n-|\alpha|-M+|\beta|}
\end{aligned}
$$

Así,

$$
\begin{aligned}
\frac{\partial M}{\partial x^{\beta} \partial z^{M-|\beta|}}(0, \ldots, 0, z)= & \sum_{n=s+1}^{\infty}\left(a_{1, \beta}^{n} \frac{\partial}{\partial x_{1}}+\ldots+a_{m, \beta}^{n} \frac{\partial}{\partial x_{m}}+c_{\beta}^{n} \frac{\partial}{\partial z}\right) \\
& \frac{\beta !(n-|\beta|) !}{(n-M) !} z^{n-M}
\end{aligned}
$$

Los términos de $j_{\infty} x(0)$ juegan su papel en $j_{\infty}\left(j_{S-1}(x \circ i)\right)(0)$, por tanto son,

$$
\begin{aligned}
& \sum_{N=s+1}^{\infty} \sum_{|\beta|=0}^{s-1}\left(a_{1, \beta}^{N} \frac{\partial}{\partial x_{1}}+\ldots+a_{m, \beta}^{N} \frac{\partial}{\partial x_{m}}+c_{\beta}^{N} \frac{\partial}{\partial z}\right) x^{\beta} z^{N-|\beta|} \\
& \text { Usando } \psi^{n}: I R^{m+1} \longrightarrow I R^{m+1} \\
&\left(x_{1}, \ldots, x_{m}, z\right) \longrightarrow\left(x_{1} z^{n}, \ldots, x_{m} z^{n}, z\right)
\end{aligned}
$$

y la fórmula en Lema 3.7 , entonces, 


$$
\begin{aligned}
& \vec{x}^{n}=\frac{1}{z^{s}}\left[\sum_{i=1}^{m}\left(\frac{1}{z^{n}}\left(x_{i} \circ \psi^{n}\right)-\frac{n x_{i}}{z}\left(x_{z} \circ \psi^{n}\right)\right) \frac{\partial}{\partial x_{i}}+\left(x_{z} \circ \psi^{n}\right) \frac{\partial}{\partial z}\right] \\
& \text { Tenemos para la componente } \frac{\partial}{\partial x_{i}} \text {, } \\
& \frac{1}{z^{s n}} \frac{1}{z^{n}} \sum_{N=s+1}^{\infty} \sum_{|\beta|=0}^{s-1} a_{i, \beta}^{N} x^{\beta} z^{n|\beta|} z^{N-|\beta|}-\frac{1}{z^{s n}} \frac{n x_{i}}{z} \sum_{N=s+1}^{\infty} \sum_{|\beta|=0}^{s-1} c_{\beta}^{N} x^{\beta} z^{n|\beta|} z^{N-|\beta|}
\end{aligned}
$$

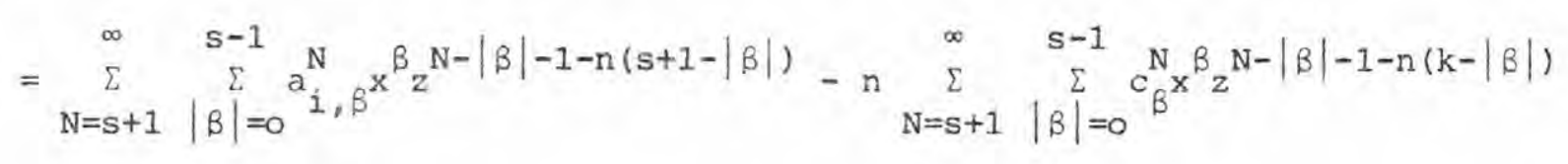

$$
\begin{aligned}
& Y \text { para la componente } \frac{\partial}{\partial z} \text { tenemos } \\
& \sum_{N=s+1}^{\infty} \sum_{|\beta|=0}^{s-1} C_{\beta}^{N} x^{\beta} z^{N-|\beta|-n(s-|\beta|)}
\end{aligned}
$$

Mirando en esta última expresión. Si esta construcción es posi ble para cada $n \in I N$, entonces $c_{B}^{N}=0, \forall N \geq s+1,0 \leq|\beta| \leq s-1$.

Así la segunda sumatoria en la expresión para la componente $\frac{\partial}{\partial x_{i}}$, es nula. Por la misma razón recién mencionada, tenemos que

$$
\begin{aligned}
& a_{i, \beta}^{N}=0 \forall N \geq s+1,0 \leq i \leq m, 0 \leq|\beta| \leq s-1 . \\
& \text { Esto establece que } j_{\infty}\left(j_{s-1}(x \circ i)\right)(0)=0
\end{aligned}
$$

(4.6) Proposición: Sea $X \in G^{m+1}$, no degenerado a lo largo del eje-z. Sea $\vec{x}$ obtenido de $x$ por $n$ blowing-ups sucesivos en 0 , en la dirección-z. Encontes $\overline{\mathrm{X}}^{\mathrm{n}}$ es no degenerado a lo largo del eje-z. 
Demostración :

Sea $\psi^{n}: I R^{m+1} \longrightarrow I R^{m+1}$

$$
\left(x_{1}, \ldots, x_{n}, z\right) \longrightarrow\left(x_{1} z^{n}, \ldots, x_{m} z^{n}, z\right)
$$

La función que determina el blowing-up $\overrightarrow{\mathrm{x}}^{n}$

Entonces para alguna funciön $g_{n}$ de clase $c^{\infty}$ tenemos que
$\vec{x}^{n}=g_{n} \bar{x}^{n} \quad y \quad x \circ \psi^{n}=\psi_{*}^{n} \circ \tilde{x}^{n}$.

Denotando por $i:[0, \infty) \longrightarrow I R^{i n+1}$

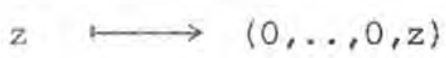

y suponiendo que $j_{\infty}\left(\bar{x}^{n} \circ i\right)(0)=0$, entonces $j_{\infty}\left(\tilde{x}^{n} 0 i\right)(0)=0 \quad y$ en consecuencia, $j_{\infty}\left(\mathrm{x} \circ \psi^{\mathrm{n}}\right.$ oi $)(0)=0$.

Pero, $\psi^{\mathrm{n}} \mathrm{i}=i$. Esto implicaría que $j_{\infty}(\mathrm{x} o \mathrm{i})(0)=0$ lo que contradice nuestra hipótesis.

(4.7) Proposición: Supongamos que $X \in G^{m+1}$ es no-degenerado a lo largo del eje-z.

Entonces es imposible que exista una sucesión infinita $\left(\bar{x}^{n}, 0, s_{n}\right)_{n \in I N}$ de Blowing-up de $x$ en 0 , en la dirección-z con $n \geq 1 ; s_{n} \geq 1$.

\section{Demostración :}

Supongamos que existe tal sucesión.

Entonces existen enteros $s \geq 1$ y $N \geq 1$ tal que $\forall n \geq 0, \bar{x}^{N+n}$

tiene grado de degeneración $\mathrm{s}$.

En consecuencia, de la proposición (4.4) $j_{\infty}\left(j_{s-1}\left(\vec{x}^{N} \circ i\right)(0)=0\right.$,

lo cual contradice la proposición (4.6).

Esta última Proposición completa la Demostración del TEOREMA, enunciado al comienzo de esta sección. 
5. BIBLIOGRAFIA.

[BO] BONCKAERT, Patrick: Smooth Invariant Curves of Singularities of vec tor Fields on $\mathrm{IR}^{3}$.

Universiteit Antwerpen. Departement WiskundeInformatica - Wilrijk, 1984.

[Du] DUMORTIER, Freddy : Singularities of Vector Fields. Monografías de Matemáticas. IMPA, RJ. 1978.

[Ta] TAKENS, Floris : Singularities of Vector Fields. Publ. Math. IHES 43 (1974). 